(setq sg29 '(1 111 1)

(setq ig8 '((sg29)))

(setq sg30'( $\left.\begin{array}{llll}1 & 1 & 1 & 1\end{array}\right)$

(setq ig9 '((sg30)))

(setq sg31' $\left.\left(\begin{array}{llll}1 & 1 & 1 & 1\end{array}\right)\right)$

(setq ig10 '((sg31)))

(setq sg32 '(1 11111$)$ )

(setq ig11 '((sg32)))

(setq tg2 '((ig5) (ig6) (ig7) (ig8) (ig9) (ig10) (ig11)))

(setq tsr '((tg1) (tg2)))

\section{Rules for automated performance of ensemble music}

Johan Sundberg, Anders Friberg and Lars Frydén
Department of Speech Communication and Music Acoustics, KTH (Royal Institute of Technology), Stockholm, Sweden

Recently developed parts of a computer program are presented that contain a rule system which automatically converts music scores to musical performance, and which, in a sense, can be can be regarded as a model of a musically gifted player. The development of the rule system has followed the aly according to the sugger of ensembleplaying also tuning, in performance of ensemble music are evaluated by a listening panel of professional musicians. Further support for the notion of melodic charge, previously introduced and playing a promir with fine tuning of intervals.

KEYWORDS music performance, timing, synchronization, tuning

\section{Introduction}

It is a well-known fact that musicians do not replicate in detail the music score. The score merely serves as a nominal description of the music requiring an interpretation, and this interpretation is signalled to the listener in terms of significant deviations from the nominal score which consequently seem musically important and meaningful.

Here we will present some recent results from a project where an analysis-by-synthesis strategy is applied to music performance. Numerous reports have already been published on this project (e.g., Friberg \& Sundberg, 1986; Friberg, Frydén, Bodin \& Sundberg, 1988; Sundberg \& Frydén, 1985; Sundberg, Askenfelt, \& Frydén, 1983a; Sundberg, Frydén, \& Askenfelt, 1983b; Thompson, Friberg, Frydén, \& Sundberg, 1986). The scenario is a highly skilled music teacher teaching the computer how to perform in a musically acceptable manner. Thus, a basic idea in this project is to make use of a musician's intuition and 
musical ideas as revealed by introspection and as developed over a long period of time through his experience of teaching.

The analysis-by-synthesis strategy for analyzing music performance complements the method of collecting measurements from actual performances, which has been applied in most investigations (see e.g., Bengtsson \& Gabrielsson, 1977; Clarke, 1985; Edlund, 1985; Gabrielsson, 1987; Shaffer, 1981; Sloboda, 1983; Todd, 1985). The limitations and advantages of these two methods have been discussed extensively elsewhere (Thompson \& al., 1986). Analysis-by-synthesis has been much less used (Clynes, 1983). There are certainly advantages and limitations associated with both these methods (Gabrielsson, 1985). An important advantage is that even those elements of music performance that are used only occasionally (and are hence difficult to find by means of a statistical processing of measurement data) can be revealed and analyzed. Moreover, if there are many reasons to shorten a note, it is a sizeable task to identify the various reasons by measuring performances. It is interesting that in performance research based on measurements, much more attention has been paid to regularly recurring events, such as the duration pattern of a bar, while expressive effects not affiliated with the bar unit have been largely neglected. In any event, there seem to be certain important advantages associated with the analysis-by-synthesis strategy as applied to music performance, and we have found it worthwhile to exploit these advantages.

In previous experiments, we have dealt with the performance of one part melodies and excerpts. Several rules have been formulated and tested, and when one or more rules have been applied, musically experienced judges report that the musical quality of the performance increases (Thompson et al., 1986).

It seems certain that these rules must also apply in ensemble music in one way or another. In the following, we will first mention some rules that seemed to be of particular relevance in an attempt to synthesize performances of ensemble music. Then, two basic aspects of ensemble playing will be considered.

(1) How is temporal coordination achieved?

(2) What principles are used for tuning chords?

Most of the background of the first question has been descibed above already, the deviations from the nominal durations of the notes seem essential in music performance but will differ between different voices in a piece of music. We may thus ask whose timetable is accepted by the others, and at which places in the piece all the musicians synchronize their note onsets perfectly.

The background to the second question deserves more detailed comments. Tuning has been a classical concern in music theory. Reviews of measurements of tuning in performance have been offered by Ward (1970) and by Sundberg (1982), and, more recently, experimental work has been reported on perceptual aspects of tuning (Hall \& Hess, 1984; Rasch, 1985; Vos, 1986). Theoretically, several tuning recipes compete.
One candidate is just tuning. It implies that all chords are played beat-free, which is often considered to be an advantage. This means that the fundamental frequencies in all simultaneously sounding major triads tuned to the frequency ratio 4:5:6, if instruments generating tones with harmonic spectra are used. An alternative is equally tempered tuning. In it, the octave is split into twelve equally wide intervals all having the frequency ratio $1: 2^{1 / 12}$.

Just tuning is sometimes referred to as pure. However, in the present article, the term pure will be used for "sounding appropriately tuned" or "perfectly in tune". As will be shown, this is sometimes quite different from being in accordance with just tuning.

Figure 1 summarizes measurements made on various types of musical performance. The interval sizes have been normalised, such that the data show the deviations in cents from what the interval is in the equally tempered scale. It can be seen that just tuning is far from universally applied in music performance. Rather, the intervals are generally played wider than just, except for the minor second which is played considerably narrower than just.

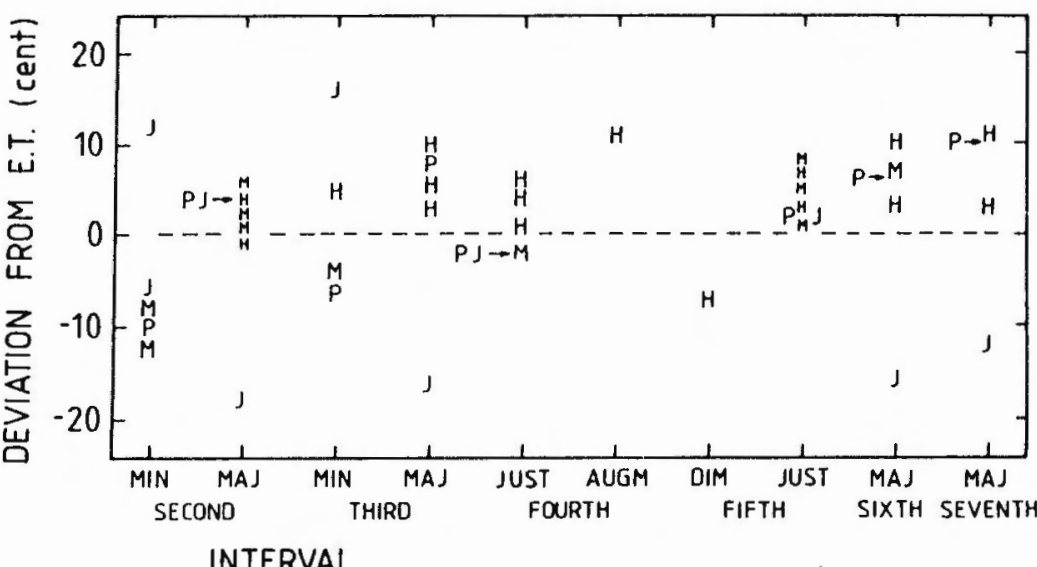

Figure 1 Mean interval sizes observed in solo $(M)$ and ensemble $(H)$ performances. J and $P$ show the corresponding values in just and Pythagorean tuning. Data adapted after Ward (1970).

In ensemble music performances, most musicians are free to decide themselves the exact fundamental frequency of the notes they are playing. This allows context-dependent intonation. Thus, they can adjust 
the frequency of each note such that each chord receives optimal tuning or such that each melodic interval is optimally sized. According to general opinion among musicians, this context-dependent tuning of each note is essential in ensemble performances. For instance, all professional players of bowed instruments would agree that they have to move their finge position if a note is changed from $\mathrm{F}$ sharp to $\mathrm{G}$ flat, and Shackford (1961 1962a; 1962b) found these two pitches to be clearly differentiated in string music performance.

In his survey of data on tuning in performance, Ward (1970) found that most intervals except the minor second are played wider than in the equally tempered scale. However, in an investigation of chord intonation in barbershop singing, several intervals were found that were narrower than in the equally tempered scale (Hagerman \& Sundberg, 1982). Furthermore, within a single note, the fundamental frequency is sometimes observed to change, apparently from one value to another (Sundberg, 1982). This suggests that the fundamental frequency averaged over an entire note may be misleading.

Ideal melodic tuning is sometimes incompatible with the demand to play beat-free chords. Thus, the melodically desirable narrow minor second often conflicts with the demands of just tuning. Let us take the progression dominant to tonic as an example. Melodically, it normally contains a minor second from the third of the dominant to the root of the tonic. Using instruments with harmonic spectra, a beat free third requires the fundamental frequency ratio of 4:5. This third is 16 cents flatter than the corresponding value in the equally tempered scale. Consequently, if the third of the dominant chord is tuned beat-free, the semitone step up to the root of the tonic will be 16 cents wider than in the equally tempered tuning. Thus, there is a conflict between what is perceived as optimal harmonically and melodically. Therefore, a third tuning scheme must be postulated. Henceforth, though as yet poorly defined, the tuning that yields intervals sounding melodically pure will be referred to as melodic tuning.

In any event, it can be assumed that the equally tempered scale, though used in some keyboard instruments, cannot a priori be regarded as the only possible solution to the tuning problem in ensemble performance. The details of the musicians' tuning strategies are largely unknown, and a primary task was therefore to look for better tuning and intonation alternatives.

\section{Procedure}

Figure 2 gives an overview of the strategy. The input is the musical score, which, voice by voice, is written into a Macintosh microcomputer. This input can be complemented by various details of interpretation, such as phrase and subphrase boundaries, harmonies, ties, etc. The input is then processed by a program (called RULLE), which automatically computes the performance and converts it into control signals for a synthesizer, using the MIDI standard. The synthesizer used is mostly a specially constructed machine, SISYPHOS, a sampler-type machine that reads previously stored wave forms at a speed determined by the fundamental frequency. One advantage with this device is that fundamental frequency can be controlled with an accuracy of $+1-0.85$ cent.

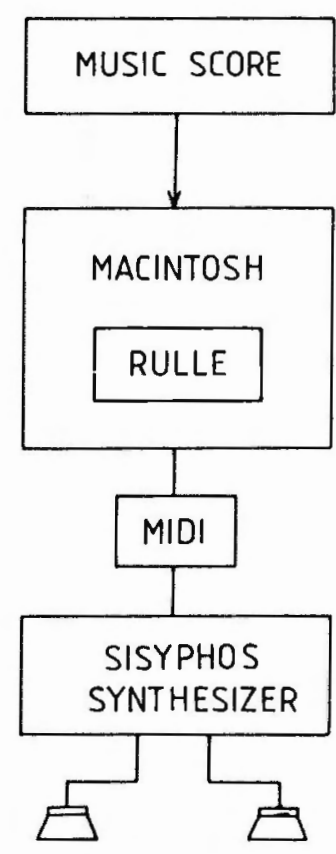

MUSIC?

Figure 2 Block scheme showing the analysis-by-synthesis strategy used in the present project. The score is written into a Macintosh microcomputer, in which the RULLE program cojverts it into control signals in a MIDI format, which are sent to a synthesizer.

Context-dependent performance rules (Friberg \& Sundberg, 1986) are introduced into the RULLE program, which is written in the Le_Lisp computer language. These rules shorten and lengthen the durations of single notes, increase or decrease their sound level, perform fine tuning of pitch, generate crescendos and decrescendos, and distribute markers 
at phrase and subphrase endings as defined in the input notation. The program allows the testing of the musical effects of each rule on the performance. In this way, the effect on performance of every single rule can be tested, demonstrated, and assessed.

In most cases, the effects of the rules are quite clearly demonstrated by this experimental setup, and only exceptionally there has been any disagreement between the authors as to whether or not a rule improved a performance. Nevertheless, in order to evaluate the various rules formally, we have had a panel of 2 professional violin players and 8 advanced music conservatory students to assess the effects. In this experiment, the subjects listened to different computer generated renderings. Their task was to rate the musical quality of the performance with a number between 0 and 7,0 being poorest and 7 being the best.

\section{Results}

\section{A. Rules for monophonic music performance}

Melodic charge

Musical notes do not form a society of equality. On the contrary, some notes are remarkable or unexpected, while others are more predictable. It seems necessary to take this into account during playing. Assume, for example, that the note of $C$ sharp occurs in a $C$ major tonality, which is a remarkable event in traditional harmony. If the player does not stress this remarkable note when playing, the performance sounds musically insensitive at this point. We have arrived at the following method for marking a note's remarkableness.

Each note is assigned a value reflecting its melodic charge. The basis of this concept is borrowed from the circle of fifths, as illustrated in Figure 3 : a note's melodic charge reflects its distance along the circle of fifths from the root of the prevailing chord. Note, however, that the distribution of melodic charge is assymmetrical around the circle; the numbers on the subdominant (left) side of the circle are negative and greater as compared to the numbers on the opposite side, which are positive. For example, three fifth steps down from the root correspond to a melodic charge of -4.5 , while three fifths steps up correspond to the charge value of 3.0. In previous reports on this project, the absolute value, only, of the melodic charge has been used. Unless otherwise stated, the term melodic charge will henceforth be used for the absolute value of the melodic charge, as before.

While the concept of melodic charge may seem farfetched, the usefulness of the circle of fifths to describe in a compact form musically relevant aspects of pitches in traditional tonal music is well-known. As shown in Figure 4, melodic charge shows a significant correlation with the mean probe tone ratings for the notes in the major scale, that Krumhansl \& Kessler (1982) reported from an experiment whose listeners rated how well different notes served as a continuation of a reference

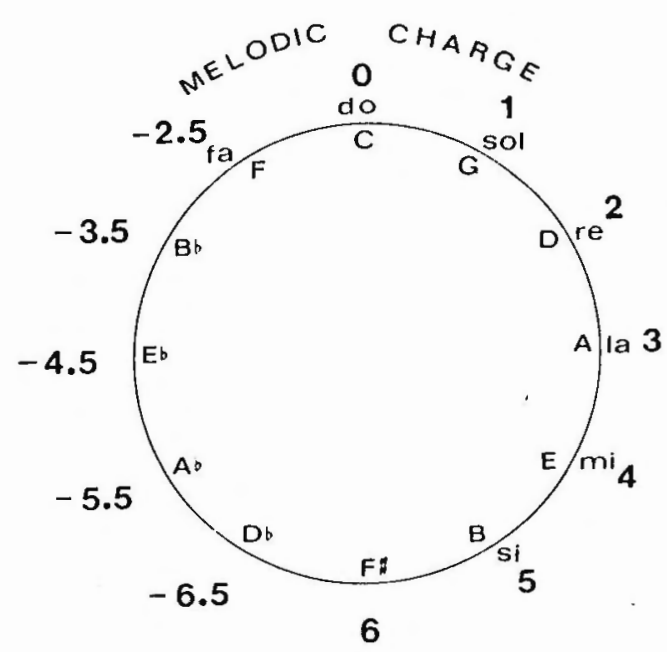

Figure 3 Definition of melodic charge of the scale tones in tonal music by means of the circle of fifths. Note that the values are asymmetrically distributed around the reference which is the root of the prevailing chord, and that the charge is negative in the left (subdominant) hal of the circle.
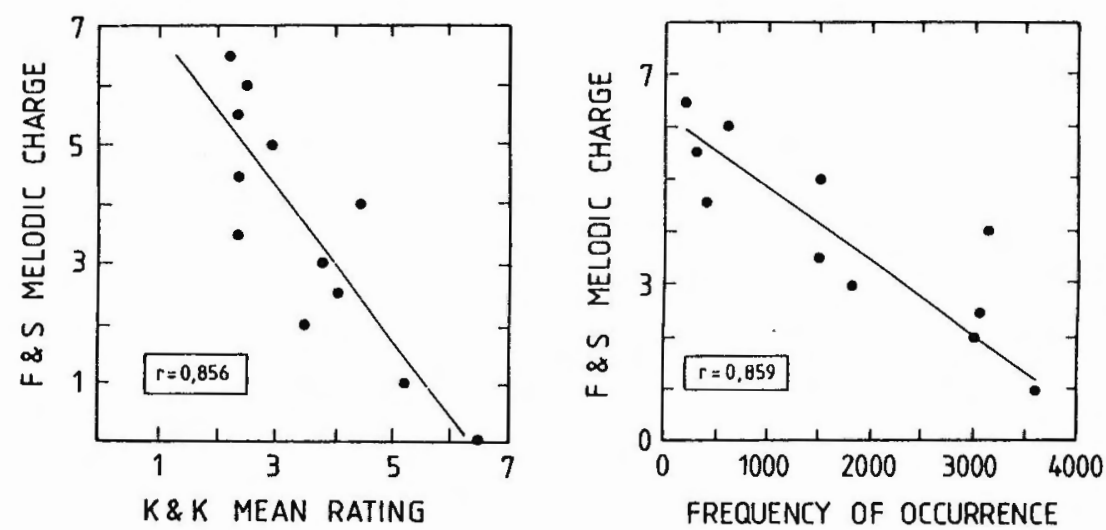

Figure 4 Correlation of melodic charge with (left) the probe tone ratings for the tones in the major scale according to Krumhansl \& Kessler (1982) and (right) the frequency of occurrence of the various scale tones in Schubert themes, as reported by Knopoff \& Hutchinson (1983). 
scale (Sundberg \& Frydén, 1987). Also, as shown in the same figure, melodic charge clearly correlates with the frequency of occurrence of the various scale tones in Schubert themes, as reported by Knopoff \& Hutchinson (1983). This suggests an interpretation of melodic charge: assuming that rarely occurring notes are remarkable, a note's melodic charge may reflect how remarkable it is.

According to our rules, melodic charge is signalled in three different ways in a performance: (1) by a lengthening of the note's duration by an amount equal to $2 / 3$ of the melodic charge, in msec; (2) by an increase of the note's sound level by an amount of $\mathrm{dB}$ equal to 0.2 times the melodic charge, and then the sound level differences between adjacent notes is smoothed; and (3) by an increase of the note's vibrato extent by 0.03 times the melodic charge, in percent. The effect is illustrated in Figure 5.
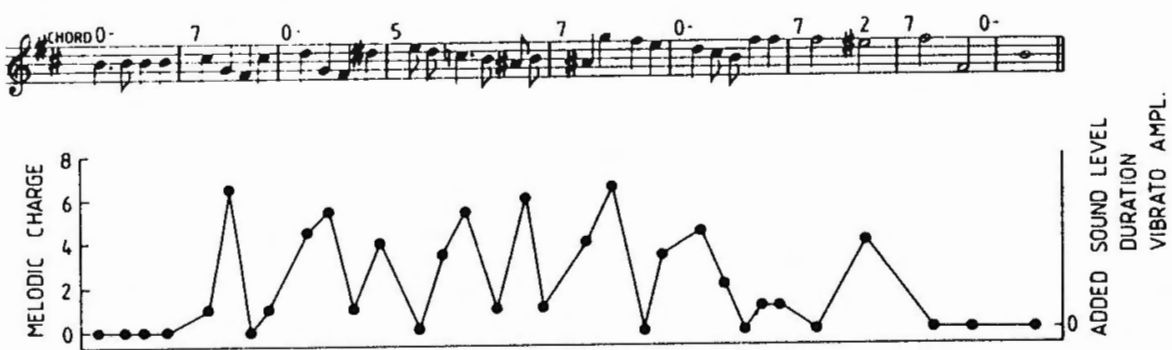

Figure 5 The effect of melodic charge on performance according to the rule system: (1) lengthening of the note's duration; (2) an increase of the note's sound level; and (3) an increase of the note $s$ vibrato extent.

\section{Harmonic charge}

The principle of inequality seems to apply also to chords. For instance a $G$ major chord appearing in a $C$ major tonality is not very remarkable while an A major chord occurring in the same tonality is much more remarkable. Again, it sounds musically insensitive if a player overlooks this when playing.

We have derived a quantitative measure of the remarkableness of chords from the chord notes' melodic charges, and we have called the resulting entity harmonic charge. The harmonic charge of a chord is a weighted sum of the chord notes' melodic charges as determined with the root of the tonic as the reference: for a triad it equals the melodic charge of the root plus $2 / 3$ of the melodic charge of the third plus $1 / 3$ of the melodic charge of the fifth. The values thus obtained are then normalized such that the harmonic charge of the tonic becomes zero. Figure 6 shows the harmonic charge values for some chords.

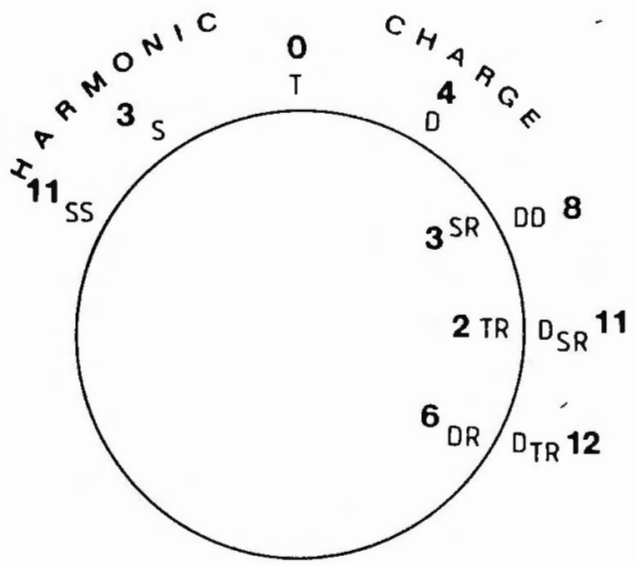
Figure 6 Harmonic charge values for some chords symbolized by their harmonic function
in tonal music: $T=$ Tonic, $S=$ Subdominant, $D=$ Dominant, $R=$ Relative. In a $C$-major context in tonal music: $\mathrm{T}=$ Tonic, $\mathrm{S}=\mathrm{Subdominant}, \mathrm{D}=\mathrm{Dominant}, \mathrm{R}=$ Relative. In a $\mathrm{C}$-major context
the chords are the following: $\mathrm{SS}=\mathrm{B}$ flat major, $\mathrm{S}=\mathrm{F}$ major, $\mathrm{T}=\mathrm{C}$ major, $\mathrm{D}=\mathrm{G}$ major, $\mathrm{DD}=\mathrm{D}$ major, $\mathrm{SR}=\mathrm{D}$ minor, $\mathrm{D}_{\mathrm{SR}}=\mathrm{A}$ major, $\mathrm{TR}=\mathrm{A}$ minor, $\mathrm{D}_{\mathrm{TR}}=\mathrm{E}$ major, $\mathrm{DR}=\mathrm{E}$ minor.

Harmonic charge may seem as farfetched as melodic charge. However, once again listeners experiences can serve as a support. Krumhansl \& Kessler (1983) presented different probe chords to listeners following a reference cadence that defined the tonality and asked the subjects to rate how well the probe chords fitted as a continuation of the reference cadence. The probe chord ratings show a significant correlation with harmonic charge, as shown in Figure 7.

According to our rules, the harmonic charge is reflected in the performance in terms of crescendos and decrescendos, as well as in minute accelerandos and rallentandos and tenutos, as illustrated in Figure 8. The sound level increases toward increases in harmonic charge and vice versa; the amplitude of the first note after each chord change is increased by a number of $\mathrm{dB}$ equal to a constant times the harmonic charge value of the new chord, and the intermediate notes are given intermediate amplitudes. In this way, crescendos and decrescendoes are created. Crescendos that are too slow are hard to detect and are avoided by delaying crescendo starts until 1.9 seconds before the chord change, whenever needed. Unlike crescendos, decrescendos start immediately after the chord change. 


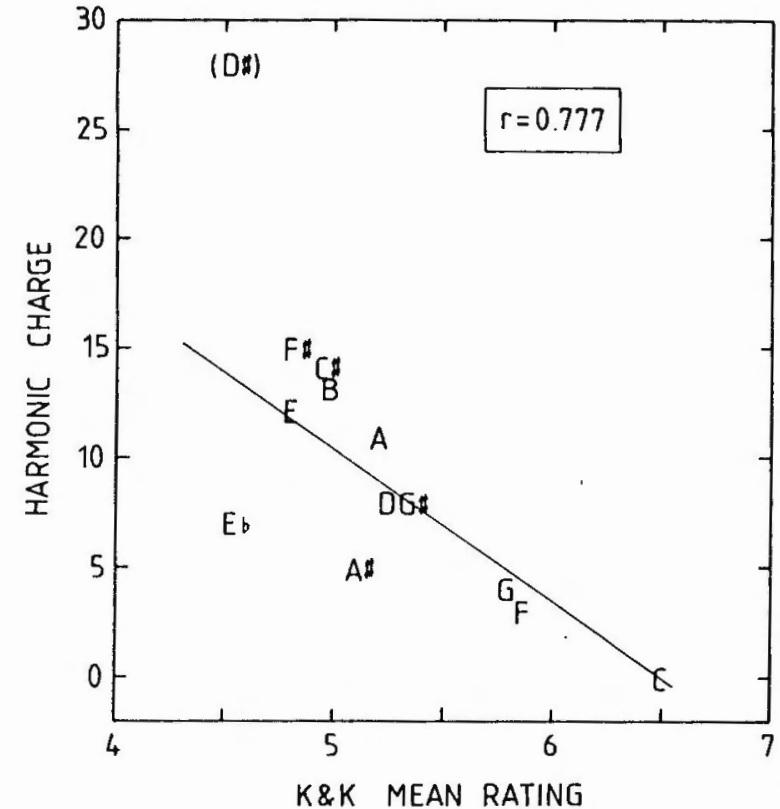

Figure 7 Harmonic charge versus the probe chord ratings reported by Krumhansl \& Kessler (1983).

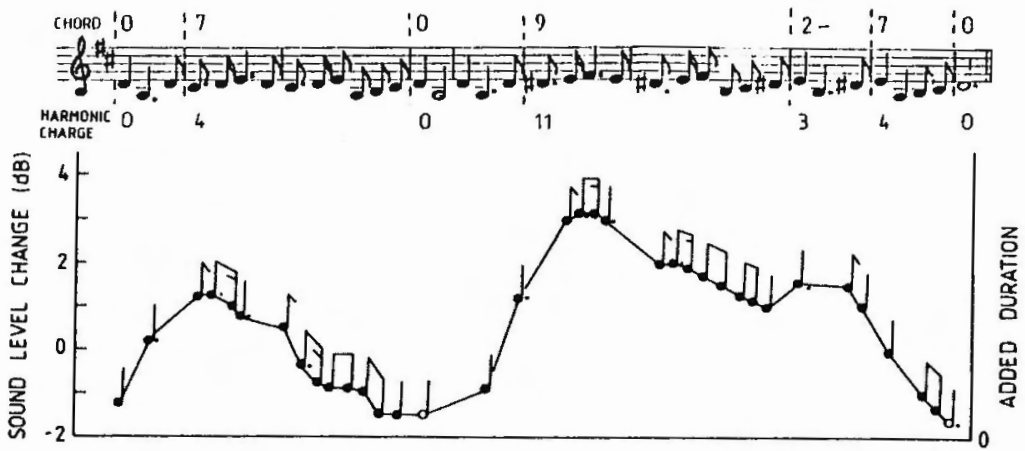

Figure 8 The effect of harmonic charge on performance according to the rule system: (1) crescendos and diminuendos; (2) concomitant tempo changes; and (3) small tenutos.
Apart from these long-term sound level variations, the harmonic charge also generates expressive variation of the notes' durations. In a crescendo, the durations of the notes are lengthened by a factor proportional to the increase in sound level, as illustrated in Figure 8. In addition, a small tenuto is added to the first note appearing together with a new, harmonically charged chord.

\section{B. Rules for ensemble music performance}

Synchronization

Two performance rules out of a total of about 20 have been presented above. As mentioned, the musical effects of these rules on performances have been approved by panels of musically experienced listeners. It seems reasonable to assume that these rules might also be valid in ensemble playing. If so, each member of an ensemble will arrive at a time pattern that is suited for his own voice or instrument. As a result a number of competing timetables will emerge in an ensemble, and some decision must be taken as to which one is going to take precedence.

One way of maintaining synchronization is that each musician should simply stick to the nominal durations. There are strong indications that this is not the normal solution in ensemble performance. We may merely point to well-known phenomena such as final ritard, tenuto and other means of expression which are clearly used in ensemble performance. We have tried out two alternative ways of achieving synchronization between voices.

One possibility is that all musicians in an ensemble introduce all the perturbations of the nominal durations that are required, but scale them such that the entire ensemble is synchronized once per bar, e.g., on the main beat. This once-per-bar principle would maintain a coordination and yet offer some leeway for individualization of the various parts. This was selected as one of the two alternatives for the listening test.

Another possibility is that everything that is vertically aligned in the score is also perfectly aligned in time and that the player, who is playing the shortest or melodically most charged note, dictates the timetable for the remainder of the ensemble. Note that this principle does not require everything to be played as nominally written in the score. This principle of synchronization on the shortest notes was selected as the second alternative for the listening experiment.

We used the following strategy in order to automatically derive a performance from the score. First, all voices were processed by all rules affecting amplitude or vibrato. Secondly, a new voice was derived from the score. This voice was built from the shortest note that appeared in the score at each moment. In those cases where there were several notes with the shortest note value, that note was selected which had the highest melodic charge. The "synchronization voice" thus obtained jumps boldly from one part to the other, as is illustrated in Figure 9. Once this synchronization voice has been constructed, it is processed by all 
performance rules that affect the duration of the notes, and all ensemble members play in synchrony with this voice.
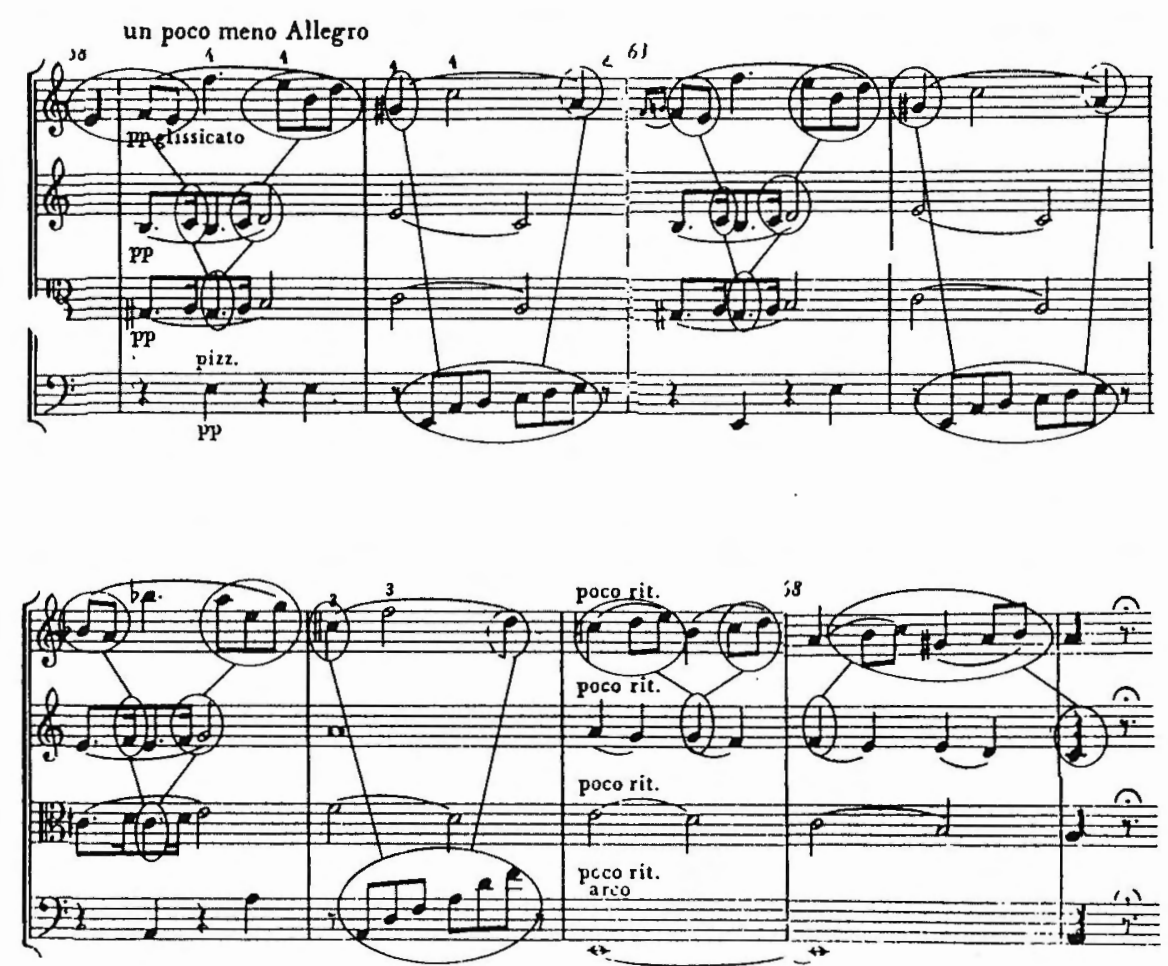

Figure 9 The excerpt from String quartet No 5, C major, second movement by Franz Berwald used in the synchronization test. The circled notes show the synchronization voice, obtained by selecting, at each moment, that voice which plays the shortest and melodically most charged notes.

Our panel of musicians rated the quality of three synthetic performances "with regard to ensemble playing, i.e., how simultaneously the musicians played". The excerpts are listed in Table 1. Each was presented three times in random order on a test tape avoiding repetitions of the same excerpt in sequence. In the examples, the synchronization was organised according to either of the two principles mentioned. The examples were presented over loudspeakers to al subjects at a comfortable listening level. The subjects gave their answers on terms of ratings between 0 and 7 on standard sheets.
Table 1 Results from the listening experiment in which professional musicians rated the quality of "togetherness" in synthesized performances of three excerpts, where the synchronization was organised according to either the "once per bar" principle or the "shortest note" principle (see text).

\begin{tabular}{|c|c|c|c|c|}
\hline \multirow{2}{*}{ Excerpt Synchronization strategy: } & \multicolumn{2}{|c|}{ "Once per bar" } & \multicolumn{2}{|c|}{ "Shortest note" } \\
\hline & $\begin{array}{l}\text { Mean } \\
\text { rating }\end{array}$ & $S D$ & Mean & $S D$ \\
\hline $\begin{array}{l}\text { H.L. Hassler: Bars } 1 \text { to } 10 \text { from Madrigal } \\
\text { "Nun fanget an" for } 5 \text { voices }\end{array}$ & 5.18 & 1.05 & 5.02 & 1.43 \\
\hline $\begin{array}{l}\text { F. Berwald: Bars } 58 \text { to } 69 \text { from } \\
\text { String Quartet No } 5, \text { C major }\end{array}$ & 2.20 & 1.19 & 4.13 & 1.33 \\
\hline $\begin{array}{l}\text { J.S. Bach: Bars } 17 \text { to } 33 \\
\text { from the Ricercar a } 6 \text { voci } \\
\text { from Musikalisches Opfer }\end{array}$ & 4.85 & 1.56 & 5.37 & 1.40 \\
\hline
\end{tabular}

The results, also shown in Table 1, showed no preference in the case of the Hassler example, where both principles yield very similar results. However, there was a clear preference for the principle of synchronisation on the shortest notes particularly for the rhythmically vivid Berwald excerpt, and less so for the Bach excerpt. According to an analysis of variance, the subjects differed significantly in their answers, and the excerpts significantly influenced the results. However, averaging over all excerpts, the principle of synchronization on the shortest notes gave significantly better ratings than the synchronization on the bar.

These results indicate a preference for the principle that all notes aligned in the score should be synchronized in the performance and that a possible timing strategy is that the player who plays the shortest and melodically most charged notes decides the timetable for the other players. This suggests that at any moment, every member of an ensemble has to listen carefully to what the other players are playing and synchronise with the timing of the most vivid musical motion, unless there is a conductor to take the responsibility for the synchronization.

\section{Tuning}

In the same listening session, different tunings were also evaluated by the panel of ten professional musicians. The two tunings tried were just tuning and equally tempered tuning. In addition, we also wanted to try melodic tuning, but as available data on melodic tuning were incomplete, we first had to carry out a preliminary tuning experiment.

In an experiment, 'we asked a very proficient violinist to tune a set of 
rising melodic intervals. The tones were produced by a synthesizer controlled from the Macintosh computer by the experimenter who changed the tuning of the interval according to the desires of the musician. Each interval was tuned at least two times. Repeated attempts to tune the same interval mostly showed a variation of a couple of cents only. His interval settings are presented in Figure 10 together with those shown previously in Figure 1.

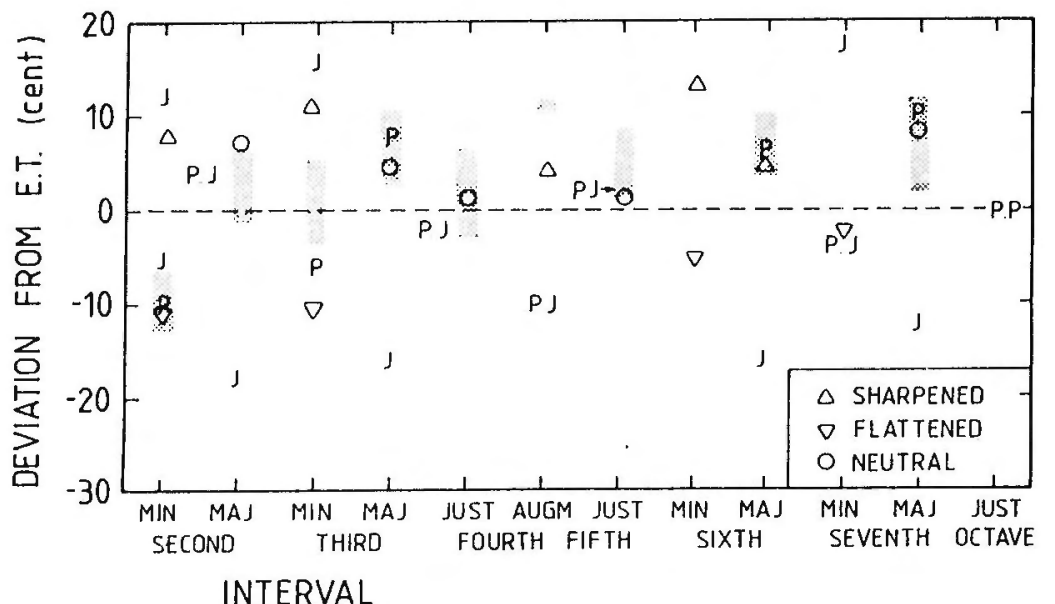

Figure 10 Deviation from equally tempered tuning of melodic interval as adjusted by a professional violin player. $\mathrm{P}$ and $\mathrm{J}$ show how Pythagorean and just tuning deviate from equally tempered. The hatched areas show the values plotted in Figure 1 representing tuning data derived from measurement on musical performance.

There is a clear tendency to increase the contrast between flattened and sharpened notes, so that flattened notes are tuned flat and sharpened notes are tuned sharp, as compared with the equally tempered tuning. In traditional harmony, a flattened note is typically followed by a falling minor second (e.g., as in the sequence $A$ flat $\rightarrow G$ ) while a sharpened note is typically followed by a rising minor second (e.g., as in the sequence $G$ sharp $\rightarrow \mathrm{A}$ ). Therefore, the tendency to increase the contrast between flattened and sharpened notes can also be seen as a tendency to decrease the size of the melodic minor second.

These interval tunings appear to be related to the signed melodic charge of the upper (target) note of the interval. Figure 11 shows the settings, expressed as deviations from just tuning (left) and from equally tempered tuning (right). In both graphs, the settings are given as a function of the signed melodic charge of the target note of the interval, assuming the lower note of the interval as the reference. The pitch symbols refer to the names of the target note of the interval, using $\mathrm{C}$ as the lower reference note. Intervals "aiming" upward, i.e., potentially followed by a rising semitone, e.g., a D sharp, are symbolized by sharps, and intervals "aiming" downward in the same sense, e.g. and E flat, by flats.
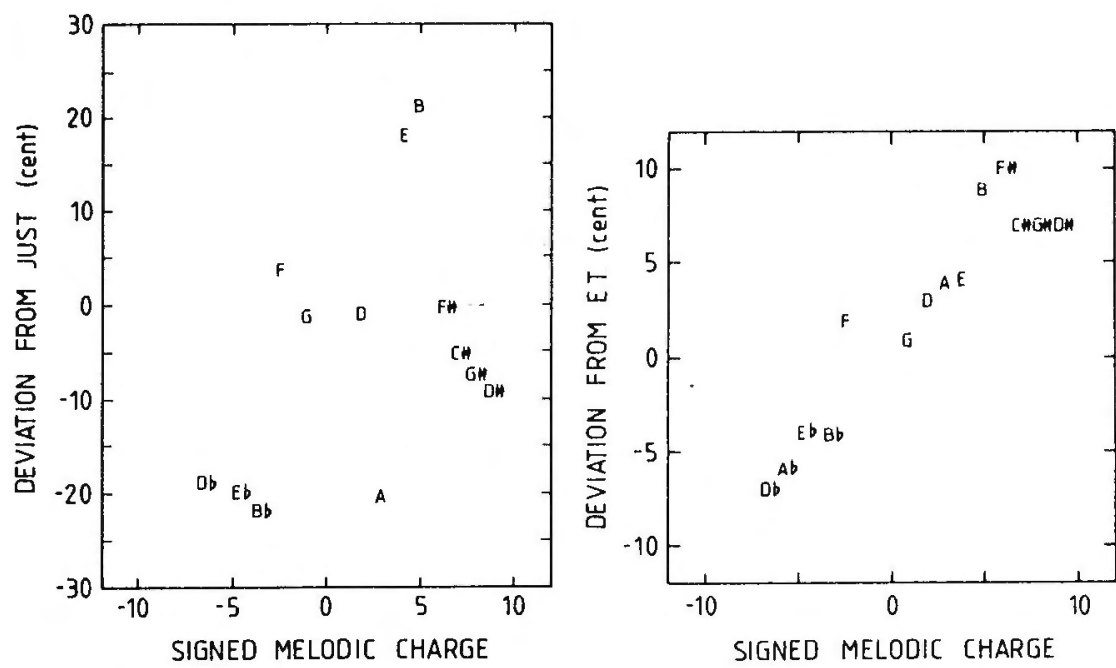

Figure 11 Deviation from just tuning (left) and from equally tempered tuning (right) of the melodic interval tunings shown in Figure 10 plotted as function of the notes' difference in signed melodic charge.

When expressed as deviations from just tuning, an overall tendency can be observed for the intervals to be expanded with increasing signed melodic charge. However, the scatter is considerable, and the data points are clustered in groups.

If, however, the data are expressed as deviations from the equally tempered scale, as in the right graph, an almost linear function is obtained. This result is interesting, as it suggests the possibility that the equally tempered scale may serve as a frame of reference for interval tuning in music performance practice. Thus, the equally tempered scale may not necessarily be only a mathematical construct, but may also represent a psychological reality. 
The straight line approximation derived by linear regression analysis $(r=0.924)$ yields almost just fifths, 701.4 cent wide on the dominant side of the circle of fifths, and slightly more on the subdominant side. These settings yielded a series of interval settings that we accepted as a fair approximation to melodic tuning.

Thus, in the listening test, subjects listened to three excerpts played in three different tunings: (1) just tuning, (2) equal temperament, and (3) melodic tuning. In addition, we also tried (4) a combination of just and melodic tuning. The reason was that neither melodic, nor just tuning sounded acceptable throughout. Rather, both sometimes sounded badly out of tune, though in different places in the pieces. For the fourth alternative, all successive notes were tuned in melodic tuning at the onset of the note, and after $10 \mathrm{msec}$, the tuning changed to just over a $70 \mathrm{msec}$ period according to a cosine curve.

The excerpts are listed in Table 2. They represent different styles of harmony. One consisted of the first 8 bars from Gesualdo's madrigal "Belt, poi che t'assenti" which contains frequent examples of chromatic changes of notes. The second example was a 15 bar extract by Purcell ("Fancy" in F major) which contains many instances of melodic minor seconds. The third example was a choral written in four voices by the contemporary Swedish composer Sven Erik Bäck ("Du som gick före oss". "Thou, who went ahead of us").

Table 2 Average rating and change in rating of just, melodic, and just-to-melodic tunings, as compared with equally tempered tuning.

\begin{tabular}{lllll}
\hline Tuning & Excerpt & $\begin{array}{l}\text { Mean } \\
\text { Rating }\end{array}$ & SD & $\begin{array}{l}\text { Changere } \\
\text { Eq. Temp. }\end{array}$ \\
\hline EQ. TEMP & Gesualdo & 3.5 & 1.53 & \\
& Purcell & 3.9 & 1.69 & \\
& Bäck & 4.75 & 1.14 & \\
& All examples & 4.05 & & \\
JUST & Gesualdo & 3.77 & 1.99 & +0.27 \\
& Purcell & 1.37 & 1.46 & -2.53 \\
& Bäck & 4.35 & 1.56 & -0.40 \\
MELODIC & All examples & 3.16 & & -0.89 \\
& Gesualdo & 1.63 & 1.50 & -1.87 \\
& Purcell & 3.6 & 1.94 & -0.3 \\
& Bäck & 3.55 & 1.56 & -1.2 \\
MEL-JUST & All examples & 2.93 & & -1.12 \\
& Gesualdo & 3.27 & 1.51 & -0.23 \\
& Purcell & 2.28 & 1.22 & -1.62 \\
& Bäck & 4.35 & 1.52 & -0.40 \\
& All examples & 3.30 & & -0.75 \\
\hline
\end{tabular}

Each excerpt was presented in each of the four tunings, and, as before, each example occurred three times in random order on a test tape that the subjects listened to over loudspeakers at a comfortable loudness level. The subjects' task was to rate the quality of the tuning, taking both harmony and melody into consideration, and they gave on a protocol sheet their ratings along a scale from 0 to 7,7 being the best. The mean ratings are shown in the same Table 2 .

According to an analysis of variance, all factors produced significant differences: subjects, excerpts, and tuning ( $p<0.01$, in all these cases). A major reason for this significance was the fact that the subjects answered very consistently when rating the three copies of each example.

Much to our surprise, the panel seemed to prefer the equally tempered tuning to just melodic, and just-to-melodic tuning. However, this preference was dependent on the music, as mentioned.

For the Purcell example, containing several minor second intervals, melodic tuning was almost as highly rated as equally tempered tuning, while for the Gesualdo and Bäck examples, composed in a homophonic, chordal style, just tuning was almost as highly rated as equally tempered tuning. The melodic-to-just tuning was slightly better than the just for the Purcell example and slightly better than melodic for the Gesualdo and Bäck examples.

These results offer good reasons for rejecting the hypothesis that just intonation can be regarded as an optimal general solution in ensemble performance. Also, it can be concluded that in tuning, the musical context must be taken into account; chords apparently sound better in just tuning while minor second sound better in melodic. Our melodic-to-just did not represent an alternative superior to the equally tempered tuning but still improved results slightly in cases where just and melodic sounded badly out of tune.

\section{Discussion}

The present investigation considered two aspects of ensemble performance: synchronization and tuning. As regards synchronization, the results suggest that musicians have to follow, at each moment, the timing of a crucial voice which consists of elements of various voices. This seems convincing in the sense that most musicians would agree on the necessity of invariably listening to the other players. During rehearsals, an important goal may be to learn the other voices and their possible time tables.

This rule for the synchronization of ensemble playing may need to be complemented by other, style-dependent rules. For instance, in certain contexts one instrument may lead over the others, e.g. in order to avoid masking effects (Rasch, 1978; 1979; 1981). In jazz music, certain dialects seem to exist where one voice may lead or lag as compared with a stable reference beat. In general, the existence of a rule seems to add one more 
possibility for musical expression, through violating this law.

In regard to tuning, the results show that equally tempered tuning is better than both just and melodic tuning, if applied throughout a piece.

The reason may be simple exposure to the tuning of pianos, organs, and synthesizers. It may even be that our use of a synthesizer, increased the jury's prejudices in this respect. An interesting future experiment would be to repeat our listening test using a violin sound.

The melodic-to-just tuning was not rated as highly as we had hoped. There may be several reasons for this. One is that the timbre used in our experiment was strongly periodic and had prominent high partials that exposed tuning deficiencies in terms of salient beats. Also, it seemed that the pitch transitions were too apparent. It is possible that musicians use vibrato to conceal tuning adjustments during playing. Furthermore, if melodic-to-just tuning is applied in music performance, it would constitute a source of error in fundamental frequency measurements; it is not completely evident how an average over time should be computed.

However, since a string player is free to make a context-dependent choice of fundamental frequency, it is not correct to infer that equally tempered tuning is better than the other tunings when the other players are also free to vary intonation. On the contrary, it seems likely that tuning is context-dependent, such that both the harmonic and the melodic functions of each note are taken into consideration. For instance, the musician playing the melody part in a homophonic piece might play according to melodic intonation, while the accompanying instruments, providing the underlying harmony, play in just tuning. In a predominantly polyphonic piece, on the other hand, melodic tuning may be more frequently used, e.g. for salient melodic intervals. The assumption that interval sizes are context dependent is also supported by the fact that some subjects found it difficult to give an overall rating, as they found both merits and deficiencies in most examples. In a future analysis of the context dependency of tuning, analysis-by-synthesis would offer an efficient tool.

It could be argued that the preliminary tuning experiment, on which the melodic tuning was based, used a stereotyped context, namely the interval between a note and the note preceding it. It is not clear whether these tuning data can be expected to be representative of music performance, where other contextual factors are also relevant, such as the note's position in the scale. In the tuning experiment, the major and minor intervals were tuned with an imagined context, viz., that they were followed by a rising or falling minor second. Nonetheless, a more detailed analysis of the relevance of the context to tuning would be needed.

Nevertheless, the settings for the melodic tuning seem convincing. First, there was a fair agreement between these data and averages found in actual performances. Second, the subject was mostly able to replicate his settings with a high level of accuracy. Third, his settings fitted nicely into the framework of the signed melodic charge.

The last mentioned fact is quite interesting. What does this correlation between tuning and the signed melodic charge suggest? According to our previous research, a performance gains in musical quality if melodic charge is marked. One may speculate that sharpening and flattening intervals is used as an expressive means in order to mark the melodic charge of the notes. This speculation is supported by an investigation by Makeig \& Balzano (1982) showing that differently sized octave intervals had different expressive meanings.

The fact that the musicians' tunings of the intervals clearly correlated with the melodic charge of the target note is interesting also from another point of view. Melodic charge is derived from the circle of fifths, and the same correlation coefficient was obtained if the position on the circle of fifths was used rather than the melodic charge in Figure 11. This means that melodic tuning is very similar to a theoretical construct called Pythagorean tuning. In this tuning all fifths are tuned just, or to 702 cent. In our musician subject's tuning, the mean fifth was slightly more narrow, or 701.4 cent. This minute difference may seem small but gains in importance, the greater the number of fifths there is in an interval.

Finally, the use of rules to describe musical performance should be commented on. Before discussing this problem it is necessary to distinguish between two aspects of a rule. One is the class of notes that are modified by the rules, i.e., the target notes of the rule. The other is the amount of modification that the rule generates in the target note's sound variables. This aspect will be referred to as the rule quantity.

Music is a form of communication by means of acoustic signals. This implies that there are some conventions shared by the player and the listeners. The rules offer efficient tools for describing such conventions. On the other hand, a given piece of music can be performed in a number of different ways, while the use of rules seemingly implies that there is one single performance possible.

One way of having the same rule system produce different performances is to allow variation of rule quantity. Many experiences support the assumption that this occurs in reality. We have noted on a number of occasions that introducing a new rule into the system necessitated a reduction of quantities of one or more of the existing rules. We have also noted that comparatively acceptable performances could be generated with only a small number of rules, suggesting that some rules are not compulsory; the quantity of these optional rules may then be regarded as reduced to zero.

There may also be alternative rules. For instance, emphasis is expressed in several different ways in our system: sound level, duration, and vibrato amplitude. A rule may merely be a line in the musician's and the listener's internal dictionary which translates items of musical expression, such as emphasis, phrase ending etc., into sound events, such as change in sound level, lengthening etc. This dictionary probably contains synonyms, and in selecting which synonym to use, the musician would take into account the style of the piece, among other things. On the other hand, some rules may not be style-dependent: in a recent experiment, it 
was found that rules developed for traditional tonal music clearly improved the performance also of contemporary music (Friberg et al., 1988).

\section{Acknowledgement}

This research is supported by the Bank of Sweden Tercentenary Foundation.

\section{References}

Bengtsson, I. \& Gabrielsson, A., (1977) Rhythm research in Uppsala, in Music Room Acoustics, J. Sundberg (ed.), Publications issued by the Royal Swedish Academy of Music Nr. 17, Stockholm, pp. 76-181

Clynes, M. (1983) Expressive microstructure in music, linked to living qualities, in Studies of Music Performance, J. Sundberg, (ed.) Publications issued by the Royal Swedish Academy of Music Nr. 39, Stockholm, pp. 76-181

Clarke, E. (1985) Structure and expression in rhythmic performance, Chapter 9, in Musical Structure and Cognition, P. Howell, I. Cross, \& R. West, (eds.) Academic Press, London, pp. 209-236

Corso, J.F. (1954) Unison tuning of musical instruments $I$. Acoust. Soc. Am. 26, pp. 746-750

Edlund, B. (1985) Performance and Perception of Notational Variants. Acta Univ. Upsaliensis, Studia musicol. Upsaliensia N. S. 9

Friberg, A. \& Sundberg, J. (1986) A Lisp environment for creating and applying rules for

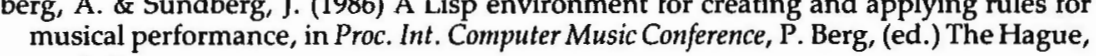
musical performance, in Proc. Int. Computer Music Conference, P. Berg, (ed.) The Hague,

pp. 1-3
Friberg, A., Frydén, L., Bodin, L-G., \& Sundberg, J. (1987) Rules for computer controlled
performance of contemporary keyboard music. Speech Transmission Laboratory Quarterly performance of contemporary kcyboard music. Speech Transmission Laboratory Quarterly Progress and Status Report, 4/1987, pp. 79-85

Gabrielsson, A. (1985) Interplay between analysis and synthesis in studies of music performance and music experience. Music Perception 3:1, pp. 59-86

Gabrielsson, A. (1987) Once again: The theme from Mozart's piano sonata in A major (K. 331), in Action and Perception in Rlythm and Music, A. Gabrielsson, (ed.) Publications issued by the Royal Swedish Academy of Music Nr. 55, Stockholm, pp. 81-104

Hagerman, B. \& Sundberg, J. (1980) Fundamental frequency adjustment in barbershop singing. J. of Research in Singing 4, pp. 3-17

Hall, D. \& Hess, J.T. (1984) Perception of musical interval tuning. Music Perception 2, pp. $166-195$

Krumhansl, C.L. \& Kessler, E.J. (1982) Tracing the dynamic changes in perceived tonal organization in spatial representation of musical keys. Psychological Review 89, pp. 334-368

Knopoff, L. \& Hutchinson, W. (1983) Entropy as a measure of style: The influence of sample length. J. of Music Theory 27, pp. 75-97

Makeig, S. \& Balzano, G. (1982) Octave tuning - two modes of perception. Paper presented at Symposium on Psychology and Acoustics of Music. Lawrence, Kansas.

Rasch, R.A. (1978) The perception of simultaneous notes such as in polyphonic music. Acustica 40, pp. 21-33

Rasch, R.A. (1979) Synchronization in unperformed ensemble music. Acustica 43, pp. 121-131

Rasch, R.A. (1981) Aspects of the Perception and Performance of Polyphonic Music. Dissertation, published by R. Rasch, Utrecht

Rasch, R.A. (1985) Perception of melodic and harmonic intonation of two-part musical fragments. Music Perception 2, pp. 441-458
Shaffer, L.H. (1981) Performances of Chopin, Bach, and Bartok: Studies in motor programming. Cognitive Psychology 13, pp. 326-376

Shackford, C. (1961, 1962a and 1962b): Some aspects of perception II II and III I. of Musc Theory 5, pp. 162-202; 6, pp. 66-90; and 6, pp. 295-303)

Sloboda, A. (1983) The Exper. Psychology 35A, pp. 377-396

Sundberg. J. (1982) In tune or not? A study of fundamental frequency in musc practise, in Tiefenstruktur der Musik. Feste A practise, in Technische Universität Berlin pp. 69-97

Sundberg. J. \& Frydén, (1987) Mp. 69-97

undberg, J. \& Frydén, L. (1987) Melodic charge and music performance, in Harmony and Tonality, J. Sundberg, (ed.), Publications issued by the Royal Swedish Academy of

Sundberic Nr. 54, Stockholm, pp. 53-58

Sundberg, J., Askenfelt, A., \& Frydén, L. (1983a) Musical performance: A synthesis by rule approach. Computer Music J. 7, pp. 37-43

Sundberg, J., Frydén, L., \& Askenfelt, A. (1983b) What tells you the player is musical? An analysis-by-synthesis study of music performance, in Studies of Music Performance, J. Sundberg, (ed.) Publications issued by the Royal Swedish Academy of Music Nr. 39, Stockholm, pp. 61-75

Sundberg, J. \& Frydén, L. (1985) Teaching a computer to play melodies musically, in Analytica, Festschrift for Ingmar Bengtsson, Publications issued by the Royal Swedish Academy of Music Nr. 47 pp. 67-76

Thompson, W.F., Friberg, A., Frydén, L., \& Sundberg, J. (1986) Evaluating rules for the synthetic performance of melodies. Speech Transmission Laboratory Quarterly Progress and Status Report 2-3, pp. 27-44; to appear, in revised form, in Psychology of Music

Todd, N. (1985) A model of expressive timing in tonal music. Music Perception 3, pp. 33-57

Vos, J. (1986) Purity ratings of tempered fifths and major thirds. Music Perception 3, Pp. 221-258

Ward, W.D. (1970) Musical perception, in Foundations of Modern Auditory Theory, J.B. Tobias, (ed.) Academic Press, New York, pp. 407-447 